\title{
Effects of poly (ethylene glycol-propylene glycol) copolymer on hemostasis and osteogenesis in a rat calvarial defect model
}

\author{
Ha-Eun Kim, Hun-Young Yoon ${ }^{1, *}$, Eun-Jin Kim², Sun-Jong Kim² \\ ${ }^{1}$ Department of Veterinary Surgery, College of Veterinary Medicine, Konkuk University, Seoul 05029, Korea \\ ${ }^{2}$ Theracion Biomedical, Seongnam 13201, Korea
}

\begin{abstract}
This study aimed to evaluate the effects of a bioabsorbable bone hemostatic agent comprising poly (ethylene glycolpropylene glycol) copolymers (PEG-PPG) on hemostasis and osteogenesis. Bilateral $3 \mathrm{~mm}$ diameter calvarial defects were created in 99 male Sprague-Dawley rats. The defects were filled with PEG-PPG or bone wax. The defects of control group were left unfilled. Virtual autopsy was performed to evaluate bioabsorption. The calvaria were subjected to x-ray microtomography (microCT) and histological examination. Bone volume fraction (BV/TV) and bone mineral density (BMD) were measured using microCT; furthermore, white blood cell count and histological examination were performed. After application of PEG-PPG and bone wax, immediate hemostasis was achieved. Autopsy revealed that PEG-PPG disappeared within $48 \mathrm{~h}$ at the application site; in contrast, bone wax remained until 12 weeks. The PEG-PPG and control groups showed significantly more osteogenesis than the bone wax group with respect to BV/TV and BMD at 3,6, and 12 weeks $(p<0.05)$. Histology revealed that the bone wax group exhibited little bone formation with inflammation. In contrast, PEG-PPG and control groups showed significantly more qualitative osteogenesis than the bone wax group $(p<0.01)$. In conclusion, PEG-PPG showed immediate hemostasis and was absorbed to allow progressive osteogenesis.
\end{abstract}

Keywords: bone, hemostasis, osteogenesis, rats, $\mathrm{x}$-ray microtomography

*Corresponding author

Hun-Young Yoon

Department of Veterinary Surgery, College of

Veterinary Medicine, Konkuk University, 120

Neungdong-ro, Gwangjin-gu, Seoul 05029,

Korea

Tel: $+82-2-450-0494$

Fax: +82-2-450-3037

E-mail: yoonh@konkuk.ac.kr

ORCID

Ha-Eun Kim

https://orcid.org/0000-0003-4918-2071

Hun-Young Yoon

https://orcid.org/0000-0001-8834-952X

Eun-Jin Kim

https://orcid.org/0000-0002-3131-4307

Sun-Jong Kim

https://orcid.org/0000-0002-3420-0938

Conflict of Interest

The authors declare no conflict of interest.

Received: October 30, 2019

Revise d: July 16, 2020

Accepted: August 4, 2020

\section{Introduction}

In orthopedic surgery, particularly in neurological surgery, hemostasis has an important effect on outcome, and uncontrolled hemorrhage may lead to postoperative complications. In general, hemostasis in soft tissue surgery is achieved via electrocautery, ligation, or manual pressure [1]; however, these methods have difficulties accomplishing hemostasis in bone. Bone hemostatic agents physically block osseous canals containing cancellous bone vessels to control bleeding on cut surfaces [2].

Bone wax is a common bone hemostatic agent that has been used for a long time. Beewax, one of its major components, was first used in 1982 [3,4]. Bone wax is composed of beewax, paraffin, and isopropyl palmitate; refined beewax, which constitutes $88 \%$ of bone wax, is an insoluble material [5]. Because of the hydrophobic nature of bone wax [5], it is not absorbed into the application site and can cause numerous side-effects [6-18].

The most common side-effect of bone wax is inhibition of osteogenesis [6,7]. The use of bone wax is prohibited in operations requiring bony fusion because it interferes with bone healing even in small amounts. Bone wax can act as a foreign body at the application site and can cause inflammation [8-12] and there is a risk of surgical-site infection [13]. Other reported side effects include poststernotomy pseudo-arthrosis [14], cervical epidural compression [15], lower extremity paralysis [16], recurrent epistaxis [17], and saphenous vein graft thrombosis [18].

Because of the complications of bone wax, alternative absorbable bone hemostatic agents have been attempted to be developed in order to avoid the use of nonresorbable bone wax. To reduce the side effects of insoluble material, new bone hemostatic agents comprising water-soluble ingredients have begun to emerge. A pluronic-based bone hemostatic agent consisting hydrophilic polyethylene glycol (PEG)-polypropylene glycol (PPG)-PEG block 
copolymer not only showed immediate hemostasis but also was absorbed within 2 days and did not interfere with bone healing in a rat femur defect model [19]. Ostene ${ }^{\circledR}$ is a commercial bone hemostatic product sold as an alkylene oxide copolymer [7]. More recently, the safety of PEG-PPG-PEG copolymer/pregelatinized starch blends was evaluated in in vitro cytotoxicity tests [20]. No adverse systemic reactions were observed in in vivo experiments using the tibia rabbit model, and the substance was absorbed within approximately 2 days and did not interfere with osteogenesis [11].

In the present study, PEG-PPG was evaluated as a new bioabsorbable bone hemostatic agent. PEG-PPG is composed of poly (ethylene glycol-co-propylene glycol) with a structure different from that of the existing PEG-PPG-PEG. The purpose of this study was to evaluate whether PEG-PPG can achieve immediate hemostasis as a bone hemostatic agent and whether it interferes with osteogenesis by remaining in the application site in a rat calvarial defect model.

\section{Materials and Methods}

\section{Animals}

Ninety-nine male Sprague-Dawley rats weighing 260-318 g, aged 7 weeks, were purchased from Korea (Orient Bio, Korea), and acclimatized to the environment for a week. They were housed in individually ventilated cages at $22 \pm$ $2^{\circ} \mathrm{C}$, with $55 \pm 5 \%$ relative humidity, with $12-\mathrm{h}$ day/12-h night cycles, and free access to water and food. This study was carried out under specific pathogen-free conditions, and was approved by the Institutional Animal Care and Use Committee of Konkuk University, Seoul, Korea (approval No. KU18006).

\section{Experimental groups}

Two types of hemostatic agents, PEG-PPG and bone wax were used in this study. PEG-PPG mainly consists of poly (ethylene glycol-co-propylene glycol). Bone wax including beewax, Vaseline, and isopropyl palmitate was purchased from Ethicon, Inc. (USA).

Ninety-nine Sprague-Dawley rats were divided into three groups and were sacrificed at 3 days, 1 week, and 2, 3, 6, and 12 weeks after surgery. Forty-five rats were used to evaluate hemostasis and bioabsorption, and autopsies were performed at 3 days, 1 week, and 2 weeks after surgery (defect number $=$ 90). To evaluate osteogenesis and inflammation, autopsies were performed in 54 rats at 3, 6, and 12 weeks after surgery (defect number $=108)$. All rats received a pair of $3 \mathrm{~mm}$ diameter defects in the calvarium, and both sides of the defects were filled with the same hemostatic agent. Bilateral defects created in the control group were left untreated.

\section{Rat calvarial defect model}

Ninety-nine 8-week-old male Sprague-Dawley rats were used to construct the rat calvarial defect model (defect number $=198$ ). Animals were placed in a small induction chamber and were anesthetized using $4 \%$ isoflurane in oxygen for $2 \mathrm{~min}$. After induction, the rats were transferred from the induction chamber to the surgical table and were maintained with $2 \%$ isoflurane via a nose hose [21].

The area to be operated on was sheared and sterilized using povidone and alcohol. A skin incision was made at approximately $2 \mathrm{~cm}$ from the nasal bone to the bregma using a No. 15 blade (Fig. 1A). After confirming the periosteum covering the calvarium, a sagittal midline periosteal incision was made, and then the periosteum was elevated laterally using the periosteal elevator. A $3 \mathrm{~mm}$ diameter defect was made on each side of the parietal bone using a trephine drill (X cube; Saeshin, Korea) (Fig. 1B). The trephine burring rate did not exceed 1,000 rpm and constant irrigation with sterile normal saline was performed to prevent thermal damage [21].

Bilateral $3 \mathrm{~mm}$ diameter defects were immediately treated with a hemostatic agent. Defects in the control group were not filled with the hemostatic agent. The periosteum was closed using simple interrupted 4-0 absorbable monofilament suture (Monocryl ${ }^{\circledR}$; Ethicon) (Fig. 1C). Skin closure was done using 4-0 non-absorbable monofilament suture

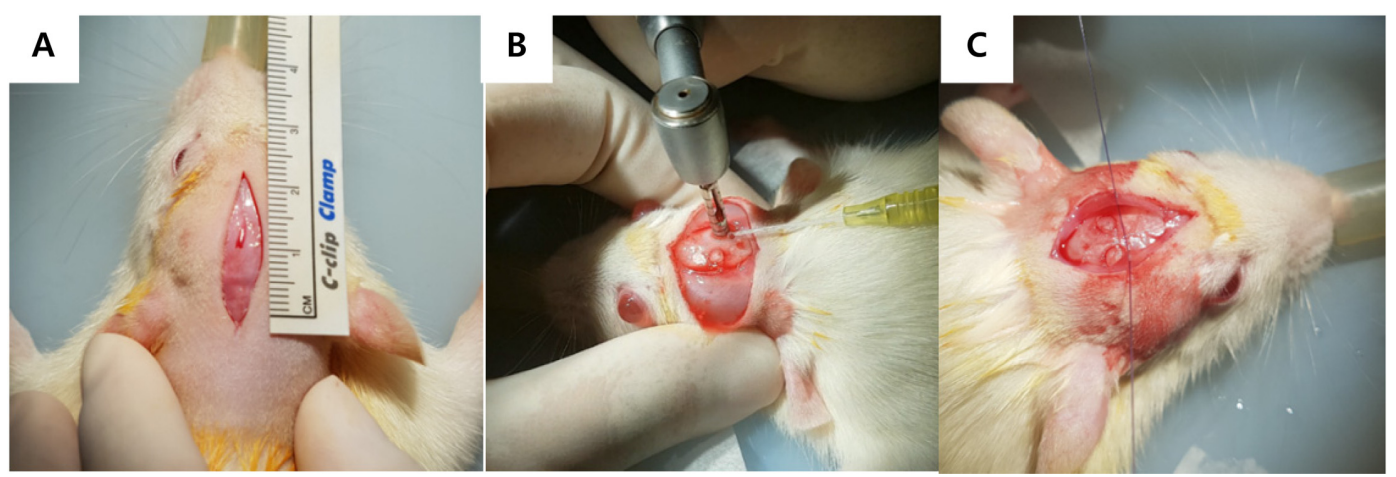

Fig. 1. The rat calvarial defect model. (A) Skin incision at approximately $2 \mathrm{~cm}$ from the nasal bone to the bregma. (B) Bilateral calvarial defect made using a $3 \mathrm{~mm}$ diameter circular trephine bur. The assistant fixes the calvarium so that it does not shake and performs saline irrigation once every $3 \mathrm{sec}$ to avoid thermal damage. (C) Suture is placed on the periosteum avoiding knots to prevent healing of the defect. 
(Dafilon $^{\circledR}$; B.Braun, Spain).

\section{Hemostasis time record}

The hemostasis time of 90 defects in the three groups was measured. The time at which hemostasis was achieved after the application of each hemostatic agent was recorded. In the negative control group, the time of hemostasis was recorded immediately after the defect was made. To assess the conditions in both the control group and the hemostatic agent application group on a similar scale, approximately $2 \mathrm{sec}$ was reduced from the time recorded in the control group when considering for analysis. Immediate hemostasis was based on $10 \mathrm{sec}$ and hemostasis was measured up to $1 \mathrm{~min}$.

\section{Gross examination for bioabsorption}

The bioabsorption of the bone hemostatic agent was evaluated by gross examination at autopsy at 3 days, 1 week, and 2 weeks after surgery (defect number $=90$ ). Autopsy confirmed the presence of any remaining hemostatic agent in the defect. For more accurate visual evaluation, calvaria containing two defects were excised to $1.5 \times 1.5 \mathrm{~cm}$ using a saw blade and were placed against a white background to confirm that there were no defects left. If a white background was seen as a defect background, it was judged that bioabsorption had occurred and the number of defects in which bioabsorption appeared was counted to compare the data between groups.

\section{X-ray microtomography (microCT) examination}

To assess osteogenesis quantitatively, microCT was performed at 3, 6, and 12 weeks postoperatively (defect number $=$ 108). The specimens were scanned using a microCT scanner (SkyScan 1173; Bruker CT, Belgium) and SkyScan 1173 control software version 1.6 (Bruker CT). The tube voltage and current were $130 \mathrm{kVp}$ and $60 \mu \mathrm{A}$, respectively. and a $1-\mathrm{mm}$ aluminum filter was used. The exposure time was $500 \mathrm{msec}$, and a pixel matrix of 2,240 $\times 2,240$ and a pixel size of $20 \mu \mathrm{m}$ were used. The rotation angle was $0.3^{\circ}$ ( $180^{\circ}$ rotation), and 800 high-resolution images in total were obtained.

Bone volume fraction (BV/TV) and bone mineral densities (BMDs) were measured. BV/TV is the relative bone volume of the newly mineralized bone for a total defect volume of $3 \mathrm{~mm}$. BMD is the density of mineralized bone as an indicator of the quality of mineralized bone.

\section{Histological analysis}

Histological examinations were performed to evaluate the quality of osteogenesis (defect number $=108$ ). For histology, each specimen was separated and fixed in $4 \%$ paraformaldehyde solution for 1 week, followed by neutral demineralization for about 1 day. The demineralized specimens were dehydrated with increasing ethanol concentrations from $70 \%$ to $100 \%$. After dehydration, specimens were clarified with xylene, embedded in paraffin, and blocks were obtained. Sections $(4 \mu \mathrm{m})$ were cut using an automated rotary microtome.
Table 1. Histological score scheme for evaluation

\begin{tabular}{ll}
\hline \hline Histological score & \\
\hline Osteogenesis & Inflammatory infiltration \\
\hline Bone formation & Granulation tissue thickness \\
$0:$ absent & 1: thick \\
1: present at the periphery & 2: absent \\
2: present centrally and at periphery & Inflammatory infiltration \\
Bone bridge & $0:$ marked \\
$0:$ absent & $1:$ mild \\
1: thin & $2:$ absent \\
2: thick & \\
Bone tissue & \\
$0:$ absent & \\
1: dense connective tissue & \\
2: bone neoformation & \\
Vascular neoformation & \\
$0:$ absent & \\
1: small & \\
2: marked & \\
\hline
\end{tabular}

Staining was carried out with hematoxylin and eosin and Masson's trichrome, and images were captured using a microscope and digital slide scanner. Morphometric measurements were obtained using Pannoramic 250 Flash III (3D HISTECH, Hungary), and images were acquired using CaseViewer version 2.0 (3D HISTECH). The quality of osteogenesis was evaluated based on the identification of the following categories: bone formation, bone bridge, bone tissue, vascular neoformation, granulation tissue thickness, inflammatory infiltration (Table 1). Scoring criteria (Table 1) were made with reference to several existing studies evaluating osteogenesis histologically [22,23].

\section{White blood cell (WBC) count}

Blood tests were performed to confirm the presence of systemic inflammation. Blood samples were collected from 18 rats at $0,3,6$, and 12 weeks after surgery (defect number $=$ 18). Blood was collected from the orbital sinus under anesthesia. WBC counts were measured using an automated hematology analyzer (Urit 2900; Diamond Diagnostics Inc., USA).

\section{Statistical analysis}

Statistical analyses were conducted using Statistical Package for the Social Sciences version 24.0 (SPSS Inc., USA) for Windows. WBC data and microCT values were expressed as means and standard deviations. Histological scores, BV/ $\mathrm{TV}, \mathrm{BMD}$, and $\mathrm{WBC}$ results were compared using one-way analysis of variance. Hemostasis and bioabsorption results were compared using Fisher's exact test. Furthermore, $p$ values of less than 0.05 were considered statistically significant.

\section{Results}

\section{Hemostatic effect}

The PEG-PPG group showed 100\% immediate hemosta- 
sis, and the bone wax group also exhibited hemostasis within $10 \mathrm{sec}$ in 29 out of 30 defects (Fig. 2). Whereas the hemostatic agent application applied group achieved immediate hemostasis, only $20 \%$ of the control group had hemostasis within $10 \mathrm{sec}$. PEG-PPG was confirmed to have an immediate hemostatic ability similar to that of conventional bone wax $(p<0.05)$ (Fig. 2).

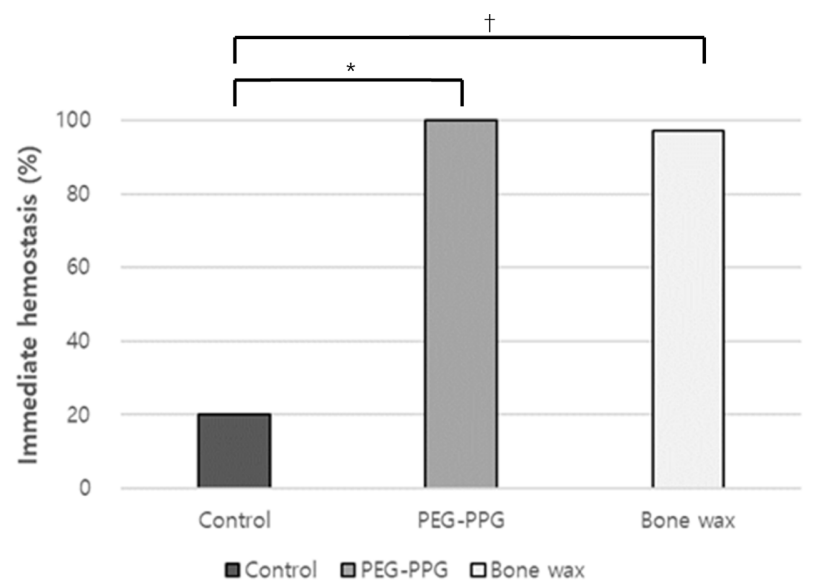

Fig. 2. Hemostatic outcomes showing the percentage of defects that achieved immediate hemostasis.

Hemostasis is considered to occur when bleeding stops within 10 sec. PEG-PPG, poly (ethylene glycol-propylene glycol) copolymers. "Compared with PEG-PPG, $p$ value $<0.05$; ${ }^{\dagger}$ Compared with bone wax, $p$ value $<0.05$.

\section{Evaluation of bioabsorption}

The bone wax remained intact in the defects, whereas the PEG-PPG was completely absorbed at 3 days after surgery (Fig. 3). All PEG-PPG defects showed bioabsorption not only at 3 days but also at 1 week and 2 weeks post-surgery, whereas the bone wax remained at the site of implantation except for 1 defect at 3 days. At 3 days after surgery, one defect in the bone wax was noted to have bioabsorption; in fact, it was not actually absorbed, but rather the implant moved to a different location. The PEG-PPG group showed significant bioabsorption compared to the bone wax group $(p<0.01)$ (Table 2).

\section{MicroCT examination of osteogenesis}

In representative $3 \mathrm{D}$ images, the bone wax group showed little bone formation in the defects at 3, 6, and 12 weeks (Fig. 4). In contrast, the control and PEG-PPG groups showed progressive osteogenesis as the new bone gradually

Table 2. Bioabsorption evaluation

\begin{tabular}{cccc}
\hline \hline Time & $\begin{array}{c}\text { Control } \\
(\mathrm{n}=10)\end{array}$ & $\begin{array}{c}\text { PEG-PPG } \\
(\mathrm{n}=10)\end{array}$ & $\begin{array}{c}\text { Bone wax } \\
(\mathrm{n}=10)\end{array}$ \\
\hline $3 \mathrm{~d}$ & $10 / 10$ & $10 / 10$ & $1^{*} / 10^{\dagger,}$ \\
$1 \mathrm{wk}$ & $10 / 10$ & $10 / 10$ & $0 / 10^{\dagger}$ \\
$2 \mathrm{wk}$ & $10 / 10$ & $10 / 10$ & $0 / 10^{\dagger}$ \\
\hline
\end{tabular}

PEG-PPG, poly (ethylene glycol-propylene glycol) copolymers. "One defect in the bone wax group was found elsewhere away from the implant site; Compared with control, $p<0.01 ;{ }^{*}$ Compared with PEG-PPG, $p<0.01$.
A

$3 \mathrm{~d}$

$1 \mathrm{wk}$
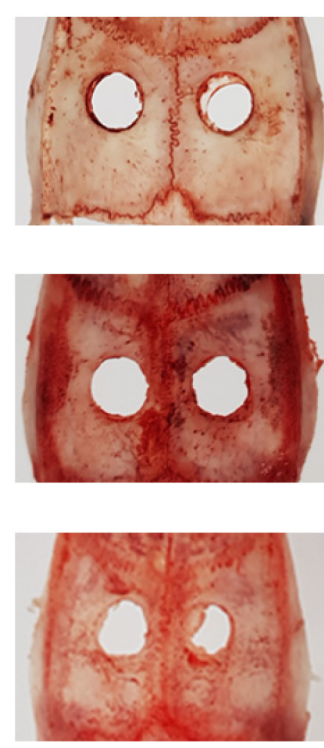

B
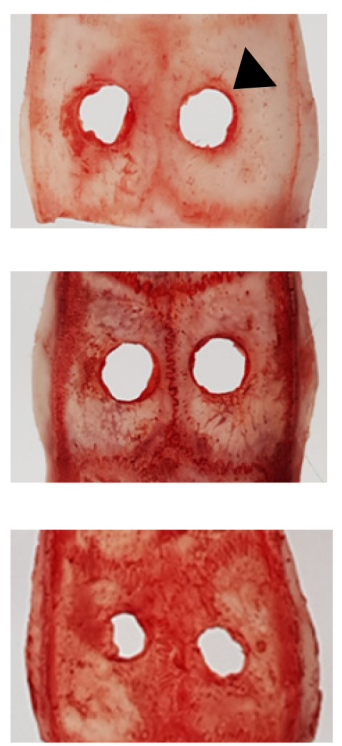

$\mathrm{C}$
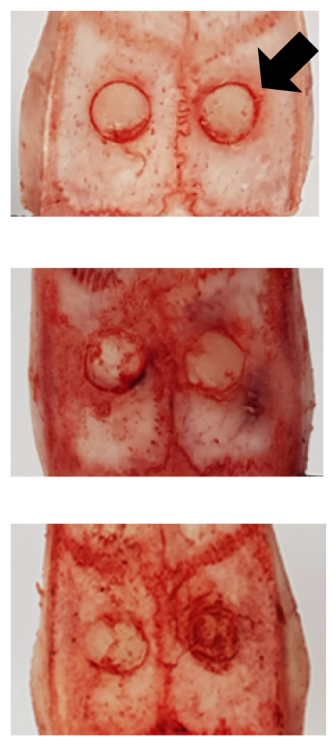

Fig. 3. Gross examination for bioabsorption. (A) Control group (B) PEG-PPG group (C) bone wax group. (A) is not filled with the hemostatic agent. In (B), there is no remaining PEG-PPG at the defect margin (black arrow head). In comparison, in the case of (C), a white background is not observed because the circular defect is filled with bone wax (black arrow). PEG-PPG, poly (ethylene glycolpropylene glycol) copolymers. 
A B

C

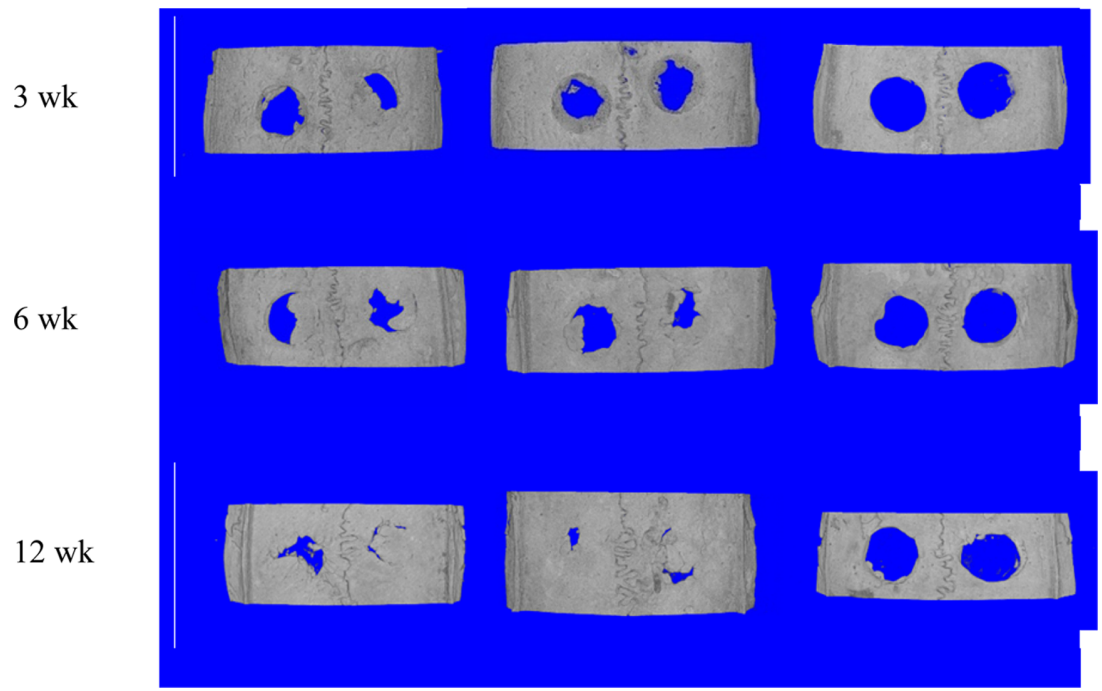

Fig. 4. Representative 3-dimensionally reconstructed microCT images of excised rat calvaria after surgery. (A) Control group (B) PEG-PPG group (C) bone wax group. (A) and (B) show progressive osteogenesis over 3, 6, and 12 weeks as new bone is gradually formed from defect margins to the centers. By contrast, (C) shows minimal bone formation at all time points. MicroCT, $\mathrm{x}$-ray microtomography; PEG-PPG, poly (ethylene glycol-propylene glycol) copolymers.

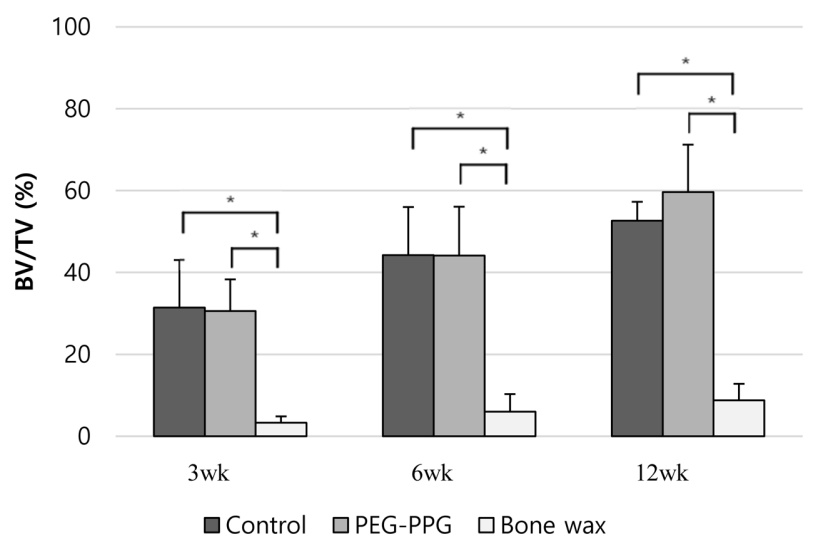

Fig. 5. Graph of BV/TV, \% measured by microCT. At all time points, the BV/TV values of the PEG-PPG and control groups are significantly higher than that of the bone wax group ( $p=0.000$ and 0.000 respectively). BV/TV, bone volume fraction; PEG-PPG, poly (ethylene glycol-propylene glycol) copolymers; microCT, x-ray microtomography. ${ }^{*} p$ value $<0.01$.

grew from the defect margin to the center (Fig. 4). The values measured through microCT were expressed as BV/TV (Fig. 5) and BMD (Fig. 6).

The bone wax group showed almost no bone healing at 3, 6 , and 12 weeks postsurgery (Fig. 4). Unlike the bone wax group, the control and PEG-PPG groups showed significant bone healing with $\mathrm{BV} / \mathrm{TV}(p<0.01)$ and $\mathrm{BMD}$ values $(p<$ $0.05)$ at all time points. A comparative graph of each value also showed significant differences between the bone wax group and the control group, and between the bone wax group and the PEG-PPG group (Figs. 5 and 6). BV/TV (Fig.

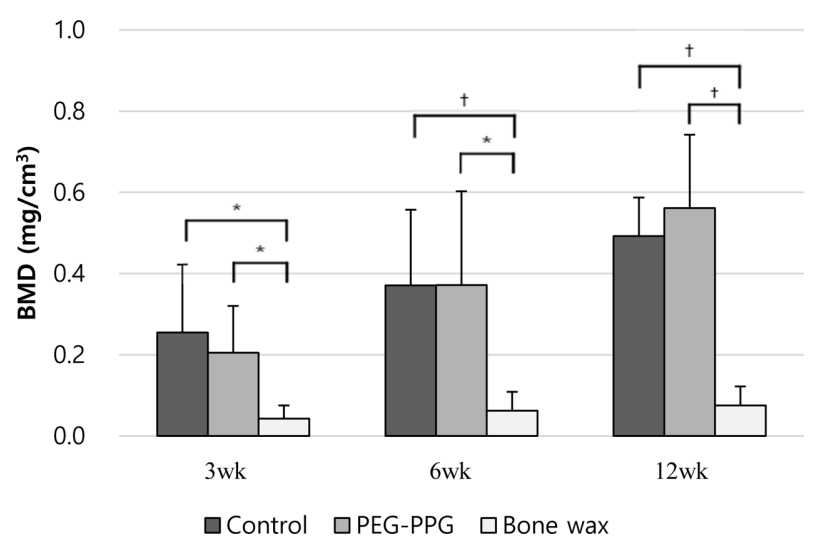

Fig. 6. Graph of BMD measured by microCT. At 3, 6, and 12 weeks, the BMD values of the PEG-PPG group are significantly higher than that of the bone wax group $(p=0.013,0.019$, and 0.000 , respectively). The control group also showed significant bone healing (BMD) compared with the bone wax group at 3 , 6 , and 12 weeks ( $p=0.029,0.003$, and 0.000 , respectively) BMD, bone mineral density; PEG-PPG, poly (ethylene glycolpropylene glycol) copolymers; microCT, x-ray microtomography. ${ }^{*} p$ value $<0.05 ; " p$ value $<0.01$.

5) and BMD values (Fig. 6) of the PEG-PPG and control groups were almost the same at all time points.

Histological analysis for osteogenesis and local inflammation

The control and PEP-PPG groups showed progressive bone formation from the defect margin to the centers, and the thickness of the newly grown bridge also became thicker 
A

$3 \mathrm{wk}$
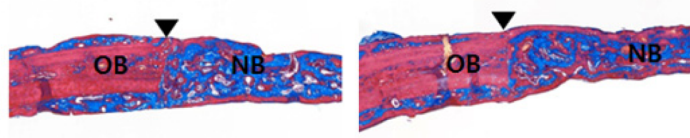

$6 \mathrm{wk}$
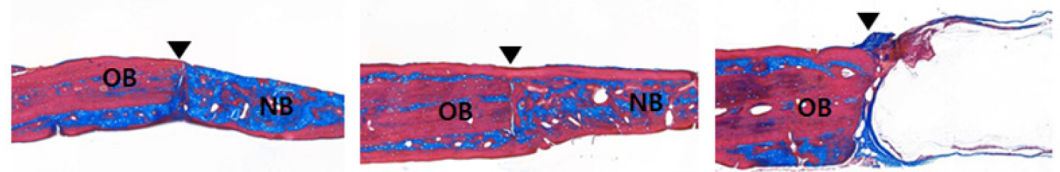

$12 \mathrm{wk}$
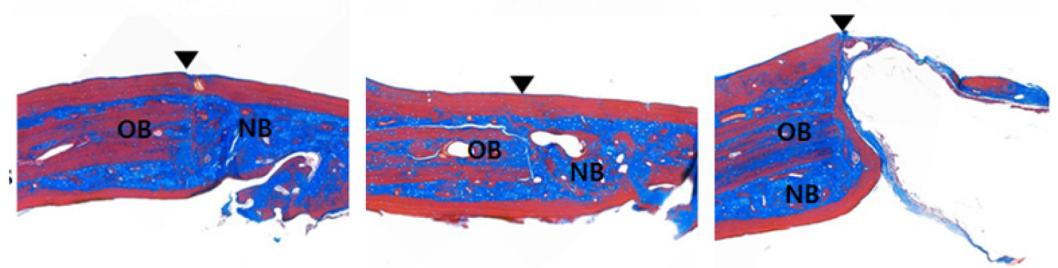

Fig. 7. Histological analysis for osteogenesis in a rat calvarial defect model. (A) control group (B) PEG-PPG group (C) bone wax group. With Masson's trichrome stain, the old bone is colored red, whereas the newly formed bone is colored blue. In (A) and (B), the new bone forms well from the defect margins (black arrow head). At 12 weeks, bone formation is almost similar to normal bone thickness. In contrast, in the case of $(\mathrm{C})$, the bone wax does not dissolve, bone formation slightly progresses, and fibrous tissue surrounding the bone wax is identified. Magnification $\times 50$. OB, old bone; NB, new bone; PEG-PPG, poly (ethylene glycol-propylene glycol) copolymers.

A

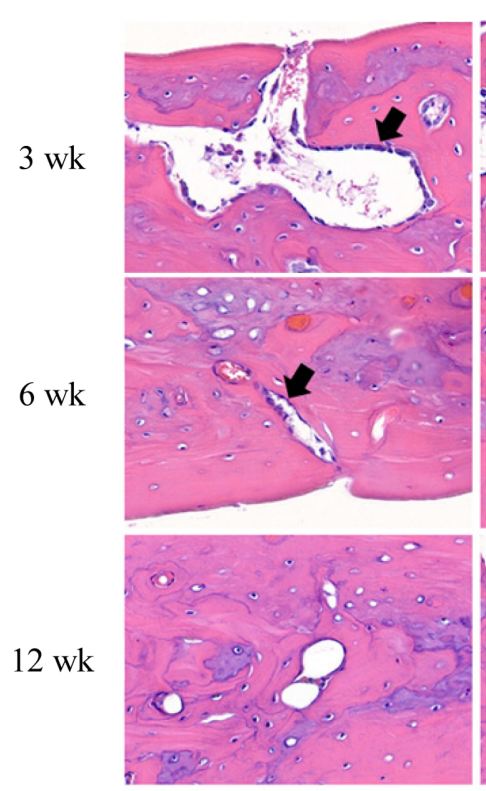

B

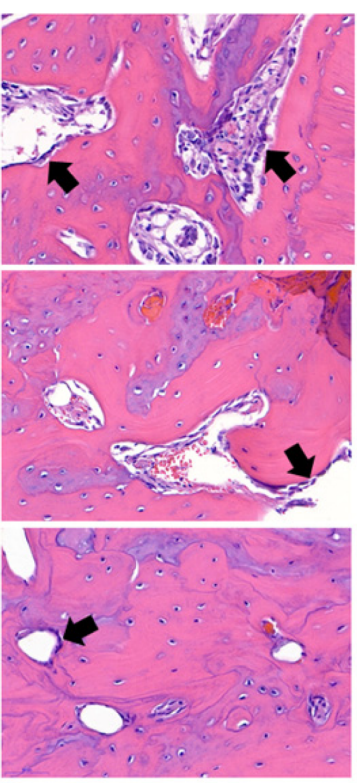

C

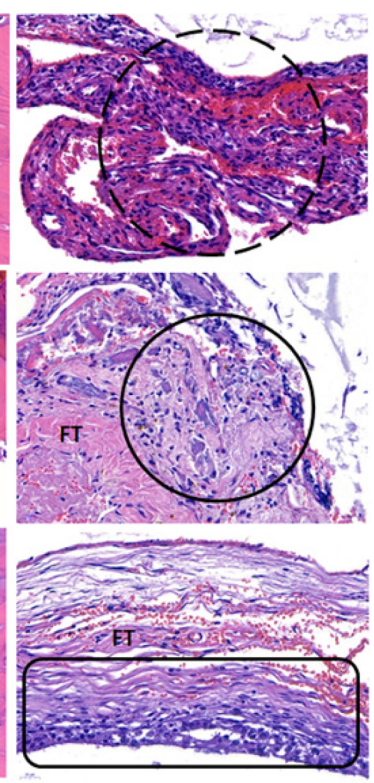

Fig. 8. Histological analysis for osteogenesis and local inflammation in a rat calvarial defect model. (A) Control group (B) PEG-PPG group (C) bone wax group. Bones that grow inside the defect margins of (A) and (B) show osteoblast activity (black arrow) with wellmineralized bones (pink area) using H\&E stain. In contrast, in the case of (C), mixed cellularity inflammation is observed at 3 weeks (circular dotted line), and inflammatory cells and fibrous tissue are observed at the contact with bone wax at 6 weeks (circular nondotted line) and 12 weeks (rectangular line). There are inflammatory infiltrates including lymphocytes, neutrophils, and macrophages in the dotted line. Magnification $\times 500$. FT, fibrous tissue; PEG-PPG, poly (ethylene glycol-propylene glycol) copolymers; H\&E, hematoxylin and eosin. 
Table 3. Histological scores of osteogenesis and inflammation

\begin{tabular}{cccc}
\hline \hline Time & Control & PEG-PPG & Bone wax \\
\hline $3 \mathrm{wk}$ & $16.33 \pm 4.59$ & $19.00 \pm 2.00$ & $6.33 \pm 3.88^{*, \dagger}$ \\
$6 \mathrm{wk}$ & $19.83 \pm 3.31$ & $20.50 \pm 1.52$ & $4.83 \pm 2.56^{*, \dagger}$ \\
$12 \mathrm{wk}$ & $19.33 \pm 2.42$ & $20.83 \pm 3.82$ & $4.00 \pm 2.76^{*, \dagger}$ \\
\hline
\end{tabular}

All data are expressed as mean \pm SD.

PEG-PPG, poly (ethylene glycol-propylene glycol) copolymers.

*Compared with control, $p<0.01$; ${ }^{*}$ Compared with PEG-PPG, $p<$ 0.01 .

over 3, 6, and 12 weeks (Fig. 7). At 12 weeks, the thicknesses of the new bone and the normal bone were similar to one another based on the defect margins in the control and PEG-PPG groups (Fig. 7). Both the control and PEG-PPG groups showed osteoblast activity at week 3 and the most prominent areas of mineralized bone at week 12 (Fig. 8). In contrast, the bone wax group had little osteogenesis (Fig. 7). Furthermore, the thickness of the granulation tissue that encapsulated the bone wax also became thicker by 3,6, and 12 weeks (Fig. 7). Furthermore, inflammatory infiltration and fibrous tissue were observed around the site of contact with the bone wax (Fig. 8). Particularly, the most marked mixed cellularity inflammation was observed at 3 weeks, and inflammatory cells such as lymphocytes, neutrophils, and macrophage were identified at 6 and 12 weeks (Fig. 8). The control and PEG-PPG groups showed significant qualitative osteogenesis compared to the bone wax group based on qualitative and local inflammation scores $(p<0.01)$ (Table 3$)$.

\section{WBC count for systemic inflammation}

The normal range of $\mathrm{WBC}$ is $2.9-15.3 \times 10^{9} / \mathrm{L}$, and the mean values at 0 week and $3,6,12$ weeks were within the normal range in all groups. All individuals were found to be at normal levels at 12 weeks, and no systemic inflammatory responses were observed in any of the groups after surgery. There were no significant differences among groups $(p>0.05)$.

\section{Discussion}

Bone wax is an effective bone hemostatic agent, providing rapid hemostasis; however, numerous side effects have been reported [7,8,10,12,13,24,25]. The most common side effect of bone wax is that it interferes with osteogenesis and induces an inflammatory reaction at the site of application [7]. Side effects of bone wax interfering with osteogenesis have been reported in various animal studies [7,24,26]. In a study using a rabbit femur defect model until up to 8 weeks, the bone wax group showed not only new bone formation but also foreign body response [7]. Even in comparison with bone wax and other hemostatic agents using the rat tibial defect model, osteogenesis inhibition and inflammatory responses were confirmed despite complete removal of the applied bone wax after $10 \mathrm{~min}$ [24]. In the present study, the side effect that bone wax interfered quantitatively and quali- tatively with osteogenesis was clearly demonstrated. Because the quantitative value of microCT was close to zero, this study showed that bone wax interfered with bone growth and mineralization. Furthermore, no histological evidence of osteoblastic activity or neovascularization was found in the defects in the bone wax group. The reason for the lack of bone healing at the bone wax implant site is the insolubility of bone wax [5]. Bone wax is composed of insoluble ingredients, including beewax that enables mechanical occlusion, resulting in bone hemostasis [5]. In contrast, beewax causes side-effects such as foreign body reactions, inflammation, and infections as well as lack of osteogenesis.

Bone wax remains indefinitely at the application site and forms a foreign body granuloma. Indeed, human studies have reported side effects of bone wax $[8,10,12,13,25]$. Because of the large amount of bone wax used for hemostasis for the reconstruction of the orbit floor defect, foreign body granuloma, and fistula formation occurred, eventually leading to a reoperation for foreign body removal [12]. Bone wax used in median sternotomy induced chronic inflammation up to 10 years as well as acute inflammation [10]. There was also a case report of a bone wax foreign body granuloma in the mastoid [8]. At the bone wax muscle implantation site, a thick band of fibrous tissue infiltrated by macrophages, giant cells, and lymphocytes was identified [7]. In the present study, histological examination confirmed mixed cellularity inflammation and fibrosis in the bone wax group. In particular, inflammatory cells including lymphocytes, neutrophils and macrophages were distributed in the area of contact with the bone wax, and fibrous encapsulation was formed around the bone wax.

Bone wax may also act as a nidus of infection. In a study in which Staphylococcus aureus was inoculated to rabbit tibiae, the bone wax group, in contrast to the soluble bone wax group, was infected, and all defects developed osteomyelitis [27]. In a human study, 6 out of 42 patients who underwent neurospinal surgery experienced surgical-site infections [13]. Bone wax causes side effects not only in the bone but also on the muscle. Bone wax was applied to the pocket of the rabbit paravertebral muscle and histological examination confirmed at 2 weeks. The increase in infection rate due to bone wax is caused by the bone wax that interferes with the ability of cancellous bone to clear the infection [28]. This appears to be due to the fact that the bone wax acts as a physical hemostatic barrier, blocking the cancellous bone and reducing bacterial clearance. Nevertheless, bone wax has been used for a long time because of its excellent hemostatic ability and relatively low cost compared with other hemostatic agents.

In the present study, bone formation continued from the edge of the defect toward the center in the control and PEGPPG groups. The calvarial healing process is affected by the bone defect margin, overlying periosteum, and underlying dura matter $[29,30]$. Thermal damage to the defect margins by burring is difficult to avoid; nevertheless, it should be 
minimized because it has an adverse influence on bone healing. In one study, ridges were removed using a rongeur to avoid thermal damage through the burring; however, a limitation of that study was that the sizes of the defects were inconsistent [6]. In the present study, in order to minimize thermal damage to the bone, the burring speed was maintained at a constant low speed of 500-600 rpm and saline irrigation was performed once every $3 \mathrm{sec}$. Furthermore, the periosteum was repositioned immediately above the defect for high osteogenic capacity [29]. In order not to interfere with bone healing at defect margins, the periosteum suture knots were kept from touching the defect. In this study, the bone wax group remained semi-permanent and not only caused brain compression, but also created a potential inflammatory source by direct contact with the underlying dura matter. In fact, when autopsied at 3, 6, and 12 weeks, most brains in the bone wax group were indented by bone wax. Although this study did not histologically or behaviorally assess the side effects of bone wax on the brain, side effects of bone wax in humans (e.g., brain compression) had been reported [12,25]. Because of the many potential side effects of bone wax, several surgeons recommend reducing the use of bone wax and, if possible, using other bone hemostats $[9,12]$.

To replace bone wax with its many potential complications, hydrophilic bone hemostatic agents were developed. PEG was first introduced as a vehicle for oxidized cellulose bone hemostatic material [26]. By comparison with bone wax, PEG/oxidized cellulose showed much less tissue irritation than bone wax and was completely absorbed within $3 \mathrm{~h}$ [26]. In another study, PEG was used as a vehicle to deliver microfibrillar collagen (MFC), a bone hemostatic agent. PEG/ MFC composites were shown to be resorbable agents, as opposed to bone wax using a rabbit calvarial defect model. PEG/MFC showed not only complete hemostasis but also a bone formation similar to that of control without hemostatic agent. However, PEG was resorbed within $8 \mathrm{~h}$, whereas MFC was degraded over a period of 2 months [31]. Unlike PEG, MFC is not only capable of inducing various allergic reactions because of its bovine origin; however, it also carries the risk of infection because of lack of bacteriostatic function [32].

Because of the side-effects of hemostatic agents with inherent biochemical hemostasis such as oxidized cellulose [33], MFC [32], and gelatin [34], new, resorbable hemostatic agents that exclude biochemical hemostatic functions and induce hemostasis only by mechanical occlusion have been studied. Pluronic is a water-soluble triblock copolymer composed of PPG and 2 PEG chains. Pluronic not only had physical properties similar to those of bone wax but also was absorbed within 24-48 h of implantation in rat femur defect models, with nearly complete reconstitution of cortical bone at 42 days postoperatively [19]. Ostene ${ }^{\circledR}$, a bone hemostat consisting of hydrophilic alkylene oxide copolymer, showed early bone healing up to 8 weeks in a rabbit femur defect model [7]. PEG-PPG-PEG copolymer/pregelatinized starch blend also showed immediate hemostasis via mechanical occlusion, safety in in vitro cytotoxicity tests [20], and significant osteogenesis without any side effects [11]. To date, there have been no cases of major side effects caused by Ostene $^{\circledR}$ and PEG-PPG-PEG in the bone. However, in one study, complications such as infiltration of inflammatory cells including macrophages and giant cells were observed at 2 weeks after transplanting Ostene ${ }^{\circledR}$ and polyethylene into muscle [7].

This present study was the first to evaluate a new bone hemostatic agent consisting of poly (ethylene glycol-co-propylene glycol). PEG-PPG mainly consists of poly (ethylene glycol-co-propylene glycol), which is a copolymer composed of PPG and PEG. This polymer material has been widely used in the pharmaceutical field as a carrier for drug delivery due to its non-ionic and biodegradable biocompatibility $[35,36]$. One study showed that PEG has the effect of preventing the early inflammatory response in the long-term auto-transplanted pig kidney [37]. Poly (ethylene glycol-copropylene glycol) has various properties according to each content of PEG and PPG. PEG-PPG is made with an optimal ratio of content to maximize hemostatic function and ease of use. More accurate results could be obtained because experiments were carried out for a long period of time (12 weeks) using a large number of experimental subjects (99 rats). Whereas majority of the bone wax group exhibited side effects, the PEG-PPG group showed significant osteogenesis without any complications similar to the control. Furthermore, PEG-PPG was superior to bone wax in terms of adhesiveness. Bone wax was not well maintained at the application site due to poor adhesion. When autopsy was performed in the bone wax group, several bone waxes were found distant from the application site. In contrast, PEG-PPG was well located at the application site because of good adhesion and stickiness.

In conclusion, PEG-PPG showed immediate hemostasis and complete absorption within $48 \mathrm{~h}$ of application in a rat calvarial defect model. PEG-PPG showed progressive osteogenesis over a 12-week period. Furthermore, PEG-PPG did not cause systemic inflammation reactions, nor local inflammation near the site of application. In contrast, bone wax interfered with bone healing and caused local inflammatory reactions. Therefore, the new bioabsorbable PEG-PPG could be used as an effective bone hemostatic agent to replace bone wax.

\section{References}

1. Lottick EA, inventor; Lottick EA, assignee. Electrocautery hemostat. United States patent US 4,370,980. 1983 Feb 1.

2. Das JM. Bone wax in neurosurgery: a review. World Neurosurg 2018;116:72-76.

3. Horsley V. Antiseptic wax. Br Med J (Clin Res Ed) 1982;1: 1165.

4. Parker R. Aural pyaemia successfully treated by removing 
putrid thrombus of jugular vein and lateral sinus. Br Med J (Clin Res Ed) 1982;1:1076-1077.

5. Sudmann B, Anfinsen OG, Bang G, Koppang R, Stølen SO, Koppang HS, Sudmann E. Assessment in rats of a new bioerodible bone-wax-like polymer. Acta Orthop Scand 1993; 64:336-339.

6. Alberius P, Klinge B, Sjögren S. Effects of bone wax on rabbit cranial bone lesions. J Craniomaxillofac Surg 1987;15: 63-67.

7. Wellisz T, Armstrong JK, Cambridge J, Fisher TC. Ostene, a new water-soluble bone hemostasis agent. J Craniofac Surg 2006; 17:420-425.

8. Low WK, Sim CS. Bone wax foreign body granuloma in the mastoid. ORL J Otorhinolaryngol Relat Spec 2002;64:38-40.

9. Ozdemir N, Gelal MF, Minoglu M, Celik L. Reactive changes of disc space and foreign body granuloma due to bone wax in lumbar spine. Neurol India 2009;57:493-496.

10. Sudmann B, Bang G, Sudmann E. Histologically verified bone wax (beeswax) granuloma after median sternotomy in 17 of 18 autopsy cases. Pathology 2006;38:138-141.

11. Suwanprateeb J, Kiertkrittikhoon S, Kintarak J, Suvannapruk $\mathrm{W}$, Thammarakcharoen F, Rukskul P. In vivo assessment of new resorbable PEG-PPG-PEG copolymer/starch bone wax in bone healing and tissue reaction of bone defect in rabbit model. J Mater Sci Mater Med 2014;25:2131-2139.

12. Wolvius EB, van der Wal KG. Bone wax as a cause of a foreign body granuloma in a cranial defect: a case report. Int J Oral Maxillofac Surg 2003;32:656-658.

13. Gibbs L, Kakis A, Weinstein P, Conte JE Jr. Bone wax as a risk factor for surgical-site infection following neurospinal surgery. Infect Control Hosp Epidemiol 2004;25:346-348.

14. Angelini GD, el-Ghamari FA, Butchart EG. Poststernotomy pseudo-arthrosis due to foreign body reaction to bone wax. Eur J Cardiothorac Surg 1987;1:129-130.

15. Cirak B, Unal O. Iatrogenic quadriplegia and bone wax. Case illustration. J Neurosurg 2000;92:248.

16. Butterworth J, Douglas-Akinwande A. Lower extremity paralysis after thoracotomy or thoracic epidural: image first, ask questions later. Anesth Analg 2007;104:201-203.

17. Tay HL, Tan LK. Surgical bone wax causing epistaxis. J Laryngol Otol 1996;110:267-268.

18. Chun PK, Virmani R, Mason TE, Johnson F. Bone wax granuloma causing saphenous vein graft thrombosis. Am Heart J 1988;115:1310-1313.

19. Wang MY, Armstrong JK, Fisher TC, Meiselman HJ, McComb GJ, Levy ML. A new, pluronic-based, bone hemostatic agent that does not impair osteogenesis. Neurosurgery 2001;49:962967.

20. Suwanprateeb J, Suvannapruk W, Thammarakcharoen F, Chokevivat W, Rukskul P. Preparation and characterization of PEG-PPG-PEG copolymer/pregelatinized starch blends for use as resorbable bone hemostatic wax. J Mater Sci Mater Med 2013;24:2881-2888.

21. Spicer PP, Kretlow JD, Young S, Jansen JA, Kasper FK, Mikos AG. Evaluation of bone regeneration using the rat critical size calvarial defect. Nat Protoc 2012;7:1918-1929.

22. de Miranda JR, Choi IGG, Moreira MS, Martins MD, Cortes ARG, Yoshimoto M. Histologic evaluation of early bone regeneration treated with simvastatin associated with lowlevel laser therapy. Int J Oral Maxillofac Implants 2019;34: 658-664.

23. Lucaciu O, Gheban D, Soritau O, Băciuţ M, Câmpian RS, Băciuţ G. Comparative assessment of bone regeneration by histometry and a histological scoring system. Rev Rom Med Lab 2015;23:31-45.

24. Ibarrola JL, Bjorenson JE, Austin BP, Gerstein H. Osseous reactions to three hemostatic agents. J Endod 1985;11:75-83.

25. Karabekır HS, Korkmaz S. Residue bone wax simulating spinal tumour: a case report. Turk Neurosurg 2010;20:524526.

26. Geary JR, Franz VK. New absorbable hemostatic bone wax; experimental and clinical studies. Ann Surg 1950;132:11281137.

27. Wellisz T, An YH, Wen X, Kang Q, Hill CM, Armstrong JK. Infection rates and healing using bone wax and a soluble polymer material. Clin Orthop Relat Res 2008;466:481-486.

28. Johnson P, Fromm D. Effects of bone wax on bacterial clearance. Surgery 1981;89:206-209.

29. Kanou M, Ueno T, Kagawa T, Fujii T, Sakata Y, Ishida N, Fukunaga J, Sugahara T. Osteogenic potential of primed periosteum graft in the rat calvarial model. Ann Plast Surg 2005;54:71-78.

30. Seo S, Heo J, Chae J, Jeon S, Kim J, Kang N, Lee K, Kang $\mathrm{K}$. Osteogenic effects on athymic rat calvarial defects by human originated cortical bone. Tissue Eng Regen Med 2009; 6:9-14.

31. Orgill DP, Ehret FW, Regan JF, Glowacki J, Mulliken JB. Polyethylene glycol/microfibrillar collagen composite as a new resorbable hemostatic bone wax. J Biomed Mater Res 1998;39:358-363.

32. Schonauer C, Tessitore E, Barbagallo G, Albanese V, Moraci A. The use of local agents: bone wax, gelatin, collagen, oxidized cellulose. Eur Spine J 2004;13 Suppl 1:S89-S96.

33. Short HD. Paraplegia associated with the use of oxidized cellulose in posterolateral thoracotomy incisions. Ann Thorac Surg 1990;50:288-289.

34. Spencer HT, Hsu JT, McDonald DR, Karlin LI. Intraoperative anaphylaxis to gelatin in topical hemostatic agents during anterior spinal fusion: a case report. Spine J 2012;12:e1-e6.

35. Pechar M, Strohalm J, Ulbrich K, Schacht E. Biodegradable drug carriers based on poly(ethylene glycol) block copolymers. Macromol Chem Phys 1997;198:1009-1020.

36. Knop K, Hoogenboom R, Fischer D, Schubert US. Poly (ethylene glycol) in drug delivery: pros and cons as well as potential alternatives. Angew Chem Int Ed Engl 2010;49: 6288-6308.

37. Hauet T, Goujon JM, Baumert H, Petit I, Carretier M, Eugene M, Vandewalle A. Polyethylene glycol reduces the inflammatory injury due to cold ischemia/reperfusion in autotransplanted pig kidneys. Kidney Int 2002;62:654-667. 